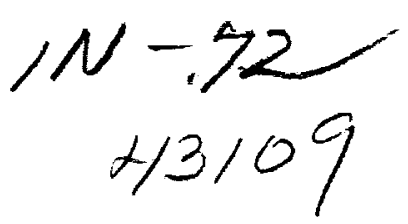

\title{
Collision Integrals and High Temperature p31 Transport Properties for N-N, O-O, and N-O
}

\author{
E. Levin \\ Harry Partridge \\ J.R. Stallcop
}

November 1989

Research Institute for Advanced Computer Science

NASA Ames Research Center

RIACS Technical Report 89.43

NASA Cooperative Agreement Number NCC 2-387

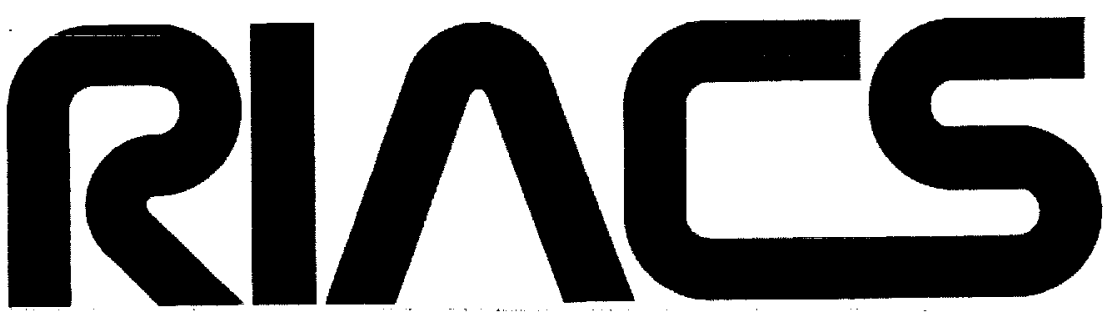

Research Institute for Advanced Computer Sclence

An Institute of the Universities Space Research Association

(NASA-CR-188907) COLLISION INTEGRALS ANO

N32-11770

HIGH TEMPERATURE TRANSPORT PROPERTIES FOR

N-N, O-O, AND N-O (Research Inst. for

Advanced computer Science) 31 P CSCL $20 \mathrm{H}$

G3/72 0043109 
$+\cdots$

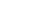




\author{
Collision Integrals and High Temperature \\ Transport Properties For N-N, O-O, and N-O \\ E. Levin* \\ Research Institute for Advanced Computer Science \\ NASA Ames Research Center, Moffett Field, California \\ Harry Partridge and J. R. Stallcop \\ Computational Chemistry Branch, Thermosciences Division \\ NASA Ames Research Center, Moffett Field, California
}

\begin{abstract}
We report accurate collision integrals for the interactions of $N\left({ }^{4} S^{\circ}\right)+N\left({ }^{4} S^{\circ}\right), O\left({ }^{3} P\right)+O\left({ }^{3} P\right)$, and $\mathrm{N}\left({ }^{4} \mathrm{~S}^{\mathrm{o}}\right)+\mathrm{O}\left({ }^{3} \mathrm{P}\right)$. These are computed from a semiclassical formulation of the scattering using the best available representations of all of the potential energy curves needed to describe the collisions. Experimental RKR curves and other accurate measured data are used where available; the results of accurate $a b$ initio electronic structure calculations are used to determine the remaining potential curves. The high-lying states are found to give the largest contributions to the collision cross sections. The nine collision integrals, needed to determine transport properties to second order, are tabulated for translational temperatures in the range $250 \mathrm{~K}$ to $100,000 \mathrm{~K}$. These results are intended to reduce the uncertainty in future predictions of the transport properties of nonequilibrium air, particularly at high temperatures. We have calculated the viscosity, thermal conductivity, diffusion coefficient, and thermal diffusion factor for a gas composed of nitrogen and oxygen atoms in thermal equilibrium. We find that the second order contribution to the transport properties is small. Graphs of these transport properties for various mixture ratios are presented for temperatures in the range $5000 \mathrm{~K}$ to $15000 \mathrm{~K}$.
\end{abstract}

\footnotetext{
* Work supported in part by Cooperative Agreement NCC 2-387 from the National Aeronautics and Space Administration (NASA) to the Universities Space Research Association (USRA).
} 


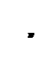

$-1$ 


\section{INTRODUCTION}

Transport properties of high temperature air are needed for upper atmosphere aerospace vehicle design and analysis studies. In particular, they are needed for a real gas analysis of the nonequilibrium flows about Aero-assisted Orbital Transfer Vehicles (AOTVs) during their aerobraking maneuvers at high altitudes and for design studies of the National Aerospace Plane (NASP).

Theoretical prediction of transport properties requires a knowledge of the potential energy curves which describe the interaction of the various colliding species of particles. Many tabulations of transport quantities have been based on incomplete potential energy information. For example, the unknown short and long range potentials of bound states have been approximated by extrapolations of analytical functions to fit the experimental data for the potential well. Moreover, the unknown potentials of higher-lying states have been estimated using crude physical models. We have found in prior studies that some interactions which were predicted to be entirely repulsive actually yield bound states; for example in the $\mathrm{N}^{+}-\mathrm{N}$ case (see Ref. 1). Since the high-lying states with high spin multiplicity usually provide the major contribution to the average collision cross sections [2,3], their potentials must be known to obtain accurate transport properties.

To reduce the uncertainty in predicted transport properties of nonequilibrium air at high temperatures, we have undertaken a systematic investigation to determine a definitive tabulation of the collision integrals of the components of air [2,3]; the transport properties of nonequilibrium air can then be obtained from these collision integrals and the relative populations of the constituents. Our approach combines an accurate determination of the unknown potentials using stateof-the-art molecular structure codes, and a calculation of the collision cross sections using computer codes that determine the scattering from the accurate potential data over the entire range of separation distances of the collision partners.

The first step in this process is an accurate large scale molecular computation of the unknown potential energy curves needed to describe the collision process. For example, in a previous 
paper [4], we computed the complete set of potential curves that correspond to the molecular states that can be formed from $\mathrm{N}^{+}-\mathrm{N}$ and $\mathrm{O}^{+}-\mathrm{O}$ when the ion-atom pairs are in their ground states. We found that the predicted potential energy curves compared well with experiment; i.e., agreed to within about $0.2 \mathrm{eV}$ with curves determined from spectroscopic and other experimental data in the region of the potential minimum. In addition, we applied the long-range potential tails of our computed results to determine $\mathrm{N}^{+}-\mathrm{N}$ and $\mathrm{O}^{+}-\mathrm{O}$ resonance charge exchange cross sections and found that the predicted results compared well with the measured data $[4,5]$. This test provided a check on the accuracy of the computed interaction energies at large separation distances.

For this work, we combined experimental data with the results of large scale structure computations to obtain the best available representations of the potential energy curves in the BomOppenheimer approximation (ignoring the spin-orbit interaction). Furthermore, the long-range potential tails are constructed to have the proper asymptotic behavior; thus ensuring that the potentials are realistic for the entire range of interatomic separation distances required for the scattering calculation. An important feature of our calculation is that a fit to the potential data by an a priori analytical function is not required to determine the collision cross sections. We have constructed a general computer code that allows the scattering to be determined from a cubic spline fit of the discrete data points which specify the potential. The application of these potential functions maintains confidence that the resultant collision cross sections are reliable. The scattering code also handles the case of multiple potential barriers [2], a condition which generally arises from the interaction of states with the same symmetry (e.g., curve crossing type interactions) and occurs for a number of states in the present calculations.

The computation of transport cross sections is based upon semiclassical approximations to a quantum mechanical description of the scattering. The scattering phase shifts are determined from JWKB approximations and a uniform approximation developed by Stallcop [6], which accounts for quantum effects when the potential energy exhibits a barrier maximum; i.e., accounts for barrier penetration and resonance scattering arising from metastable energy levels. 
Our approach avoids certain problems of a classical description of the scattering; for example, the unrealistic singularity in the classical deflection angle for orbiting energies (an artifact of classical mechanics) and should permit reliable determination of the scattering even at low collision energies or for the case of light collision partners such as hydrogen. Moreover, this semiclassical method allows the resonance charge transfer process to be included in ion-atom collisions without ad-hoc assumptions [see Ref. 2].

In the present work, we report results for $\mathrm{N}-\mathrm{N}, \mathrm{O}-\mathrm{O}$ and $\mathrm{N}-\mathrm{O}$ system interactions. Preliminary results for these systems have been reported by the authors at recent AIAA meetings [2,3]. Our tabulations of the collision integrals cover a broad range of temperatures from $250 \mathrm{~K}$ to $100,000 \mathrm{~K}$ and can be applied to determine transport properties such as viscosity, thermal conductivity, diffusion coefficient, etc. to second order. We have calculated selected transport properties of a gas composed of nitrogen and oxygen atoms in various mixture ratios to illustrate the use of the tabulated collision integrals. These results combined with the transport properties of ions and of molecules could be lused to calculate the transport properties of the real gas. The mathematical formulation of our calculation is described in Section II. In Section III, we present the details of the construction of the interaction curves used as input. The results of our calculations are shown and discussed in Section IV.

\section{METHOD}

According to Chapman-Enskog theory [7-9], the transport properties of dilute monatomic gases can be expressed in terms of the reduced transport collision integrals,

$$
\sigma^{2} \Omega_{n, s}(T)=\frac{F(n, s)}{2(k T)^{s+2}} \int_{0}^{\infty} e^{-E / k T} E^{s+1} Q_{n}(E) d E
$$

where $k$ is the Boltzmann constant, $\mathrm{T}$ is the translational temperature, and the factor

$$
F(n, s)=\frac{4(n+1)}{\pi(s+1) !\left[2 n+1-(-1)^{n}\right]}
$$


scales the collision integral to the value for scattering by a hard sphere of radius $\sigma$.

The evaluation of the collision integrals of Equation (1) requires knowledge of the collision cross sections $Q_{n}(E)$ for a broad range of values of the collision energy $E$. From quantum theory, these collision cross sections may be expressed in terms of the scattering phase shifts $\eta_{l}$, which in turn depend on the (adiabatic) interaction potentials $\mathrm{V}(\mathrm{r})$ between the colliding particles. The cross sections are calculated from:

$\mathrm{Q}_{\mathrm{n}}=\frac{4 \pi}{\mathrm{k}^{2}} \sum_{v>0}^{\mathrm{n}} \sum_{l=0}^{\infty} \mathrm{a}_{\mathrm{nv}}^{l} \sin ^{2}\left(\eta_{l+\mathrm{v}}-\eta_{l}\right)$

where $l$ is the angular momentum quantum number, $\mathrm{k}$ is the wave number, and the allowed values of $v$ are $\left\{\begin{array}{l}\text { even } \\ \text { odd }\end{array}\right\}$ according to the even or odd parity of $n$.

The coefficients $\mathrm{a}_{\mathrm{nv}}^{l}$ are determined by recursion from [10]

$(2 l+1) \mathrm{x}^{\mathrm{n}} \mathrm{P}_{l}(\mathrm{x})=\sum_{\mathrm{v}=-\mathrm{n}}^{\mathrm{n}} \mathrm{a}_{\mathrm{nv}}^{l} \mathrm{P}_{l+\mathrm{v}}(\mathrm{x})$

where $\mathrm{P}_{l}(\mathrm{x})$ are the Legendre Polynomials.

At high energies (e.g., for collision energies far above the barrier maximum), the scattering phase shifts $\eta_{l}$ are approximated by the JWKB formula,

$\frac{\eta_{j w k b}}{k}=\int_{r_{x}}^{\infty}[G(r)]^{1 / 2} d r-\int_{b}^{\infty}\left[1-\frac{b^{2}}{r^{2}}\right]^{1 / 2} d r$

where the lower limit $\mathrm{r}_{\mathrm{x}}$ of the first integral is the largest root of

$G(r) \equiv 1-\frac{V(r)}{E}-\frac{b^{2}}{r^{2}}=0$,

and $\mathrm{b}=(l+1 / 2) / \mathrm{k}$ can be identified with the classical impact parameter.

At lower energies, we apply a different method for the determination of the phase shifts. The 
uniform phase shift approximation developed by Stallcop [6] accounts for tunnelling through the barrier of the effective potential energy and also accounts for resonance scattering associated with metastable energy levels of the inner potential well and virtual energy levels above the barrier maximum (see Fig. 1).

For energy levels below the barrier,

$$
\begin{aligned}
& \eta=\eta_{j w k b}+\rho+\arctan \left\{\frac{\sqrt{1+e^{-2(I+\tau)}}-1}{\sqrt{1+e^{-2(I+\tau)}}+1} \tan (\phi+\rho)\right\} \text {, where } \\
& \rho=0.5 \arg \Gamma\left\{1 / 2+\frac{\mathrm{iI}}{\pi}\right\}-\frac{\mathrm{I}}{2 \pi} \ln \left|\frac{\mathrm{I}}{\mathrm{e} \pi}\right|, \\
& \tau=0.5 \ln \left\{\Gamma\left(1 / 2+\frac{\phi}{\pi}\right) / \sqrt{2 \pi}\right\}-\frac{\phi}{2 \pi} \ln \left|\frac{\phi}{\mathrm{e} \pi}\right|, \\
& \phi=k \int_{\mathrm{r}_{1}}^{\mathrm{r}_{2}}[\mathrm{G}(\mathrm{r})]^{1 / 2} \mathrm{dr}, \\
& \mathrm{I}=\mathrm{k} \int_{\mathrm{r}_{2}}^{\mathrm{I}_{3}}|\mathrm{G}(\mathrm{r})|^{1 / 2} \mathrm{~d} r,
\end{aligned}
$$

and $r_{1}, r_{2}, r_{3}$ are the ordered roots of $G(r)=0$.

For energy levels above the barrier, there is only one root, $r_{1}$, and $\eta$ takes the form

$$
\begin{aligned}
& \eta=\eta_{j w k b}+2 \rho-\arctan \left\{\frac{\sin 2(\phi+\rho)}{\sqrt{1+e^{-2 I}}+\cos 2(\phi+\rho)}\right\}, \text { where now, } \\
& \phi=k \int_{r_{1}}^{r_{\text {max }}}[G(r)]^{1 / 2} d r \\
& \rho=0.5 \arg \Gamma\left\{1 / 2+\frac{i I}{\pi}\right\}-\frac{I}{2 \pi} \ln \left|\frac{I}{e \pi}\right| \text {, and }
\end{aligned}
$$


$I=\frac{-\pi k}{\sqrt{2}}\left\{\frac{G}{\sqrt{\left|G^{\prime \prime}\right|}}\right\}_{r=T_{\operatorname{tax}}}$.

The quantity $r=r_{\max }$ specifies the position of the barrier maximum. The expression for $I$ is based on a local parabolic expansion near this maximum.

The above formulation was incorporated into a computer program, "SKATR" which is structured to take full advantage of the accurate interaction potentials described in Section III. The program fits a spline curve through the discrete potential data and joins smoothly with the theoretically correct specifications of the long-range interactions. For multiple potential barriers, resonance scattering is not taken into account.

\section{INTERACTION POTENTIALS}

The interaction energies (which correspond to the potential energy curves of the various states of the molecules formed from the colliding atom-atom pairs) are needed as input to determine the transport cross sections. We have combined experimental data with the results of $a b$ initio molecular structure calculations to obtain the best representation of the true interaction energies. State-of-the-art $a b$ initio calculations yield results that are in excellent agreement with the experimentally derived potentials $[11,12]$. For example, recent calculations on the bound states of $\mathrm{O}_{2}$ give dissociation energies that are accurate to within $0.03 \mathrm{eV}$ [12]. The interaction potentials employed in this work differ slightly from those reported in Ref. 3. Tabulations of the potentials are available from the authors.

Where available we have used experimental data such as the measured dissociation energy and RKR turning points [13] to represent the potential in the region of the well minimum. Ab initio molecular structure potential calculations are used to extend the experimental data to the adjoining regions by adjusting the $a b$ initio results to agree with the experimental data. Since electron exchange interactions dominate in this region (i.e., the potential energy behaves roughly like an exponential function $\mathrm{V}_{\mathrm{o}} \mathrm{e}^{-\alpha \mathrm{r}}$ ), the adjustments were made in such a way as to preserve the value 
of the shielding parameter, $\alpha$. For small $r$, where the potential curves are steep, we have shifted the $a b$ initio potentials by $\Delta r$, the difference between the calculated and measured equilibrium separation distance $r_{e}$. Since the measured and calculated values of the potential curves are in good agreement (e.g., Ref. 4), the transport cross sections are not very sensitive to the manner of adjusting the $a b$ initio results to be compatible with the measured data.

The $a b$ initio interaction curves have been extended by a proper form of the long-range potential interaction. Neutral atom interactions exhibit an induced dipole-induced dipole interaction; hence, the potential energy has the form $-1 / 2 \mathrm{C}_{6} \mathrm{r}^{-6}$. The parameter $C_{6}$ has been determined either from the $a b$ initio results at large $r$ or taken directly from other approximations to the Schroedinger equation such as perturbation expansions [14]. In addition, some of the $O-O$ interactions have a nonvanishing quadrupole-quadrupole interaction; in this case, the potential energy has a long-range term, $\mathrm{C}_{5} \mathrm{r}^{-5}$. The values of $\mathrm{C}_{5}$ have been determined from the asymptotic formulation developed by Chang [15].

We now describe in detail the source of all the potential curves. The potential wells of the strongly bound $\mathrm{X}^{\mathrm{l}} \Sigma_{\mathrm{g}}^{+}$and $\mathrm{A}^{3} \Sigma_{\mathrm{u}}^{+}$states of $\mathrm{N}_{2}$ were obtained from $\mathrm{RKR}$ and other experimental data $[13,16,17]$. Additional potential information for these states was derived from the configuration interaction studies of Ermler, McLean and Mulliken [18]. The potential curve for the $\mathrm{A}^{\prime 5} \Sigma_{\mathrm{g}}^{+}$state was obtained from recent accurate calculations by Partridge et al. [19]. The inner potential well of this state was found to be much deeper than that of previous computations [20]; this has led to assignment of the long-known, but previously unassigned, Hermann infrared bands to the transition $C^{\prime \prime 5} \Pi_{u} \rightarrow A^{\prime 5} \Sigma_{g}^{+}$[19]. Recent spectroscopic measurements by Huber and Vervloet [21] confirm this assignment and support the values of the parameters predicted from the calculated potential data. The repulsive region of the $\mathrm{X}^{1} \Sigma_{\mathrm{g}}^{+}$state was extended to small $\mathrm{r}$ using the generalized valence bond calculations of Dunning et al. [22] and Ermler et al. [23]. The ${ }^{7} \Sigma_{u}^{+}$state potential of Partridge et al. [24] has been extended to smaller $r$ by adjusting the SCF potential determined by Ferrante and Stwalley [25]. The long-range interaction energies computed in Ref. 24 strongly support the value of the dispersion coefficient deduced by Zeiss 
and Meath [14] and we have used their values for all the long-range $\mathrm{N}-\mathrm{N}$ interactions.

For oxygen-oxygen interactions, ab initio energies corresponding to the $c^{1} \Sigma_{\mathrm{u}}^{-}, \mathrm{A}^{3} \Sigma_{\mathrm{u}}^{+}, \mathrm{A}^{\prime 3} \Delta_{\mathrm{u}}$, ${ }^{1} \Pi_{g},{ }^{3} \Pi_{u}$ and ${ }^{5} \Pi_{g}$ states of $O_{2}$ were obtained from the recent calculations of Partridge, et al. [12]. Ab initio energies for the other states of $\mathrm{O}_{2}$ were taken from the FOCI results of Saxon and Liu [27]. The potential wells of the $X^{3} \Sigma_{g}^{-}, a^{1} \Delta_{g}, b^{1} \Sigma_{g}^{+}, c^{1} \Sigma_{u}^{-}, A^{\prime 3} \Delta_{u}$, and $A^{3} \Sigma_{u}^{+}$states have been determined from experimental data $[13,28-33]$. In addition, we have included corrections for some Rydberg states and shifted energies at small $\mathrm{r}$ to give better agreement with electron scattering measurements. The Rydberg character of the ${ }^{3} \Pi_{\mathrm{u}}$ state at small $\mathrm{r}$ has been taken into account using the potentials calculated by Buenker and Peyerimhoff [34] and Guberman and Dalgarno [35]. Similarly, Rydberg corrections to the ${ }^{3} \Pi_{g}$ and ${ }^{1} \Pi_{g}$ states have been taken from Saxon and Liu $[36,37]$. Furthermore, the potential curves of the ${ }^{3} \Pi_{u}$ and ${ }^{3} \Pi_{g}$ states have been shifted at small $\mathrm{r}$ to be consistent with the potentials deduced from electron scattering measurements [38-40]. The value of $\mathrm{C}_{6}$ for the $\Sigma$ states was obtained from Ref. 14; for the other states, it was deduced from the $a b$ initio energies after subtracting the contribution from the long-range quadrapole-quadrapole interaction.

The $\mathrm{N}$ - $\mathrm{O}$ potential curves have been determined primarily from $\mathrm{MRCI}+\mathrm{Q}$ calculations $[11,41$, 42]. The potential wells of the $\mathrm{X}^{2} \Pi$ and $\mathrm{a}^{4} \Pi$ states have been adjusted to agree with measured data $[13,43]$. (The spin-orbit interaction allows the $X^{2} \Pi$ state to be resolved spectroscopically into $\Pi_{3 / 2}$ and $\Pi_{1 / 2}$ components. We have not incorporated this refinement in our scattering calculations since the splitting energy is small, $\approx .014 \mathrm{eV}$ [13]. This is within the uncertainty of the molecular structure calculations.) The value of the long-range parameter $C_{6}$ for the $\Pi$ states was determined by the combining rules of Ref. 14 from their $\mathrm{N}_{2}$ and $\mathrm{O}_{2}$ data; for the $\Sigma$ states, it was calculated from the $a b$ initio energies.

The potential data obtained from the combined experimental and theoretical data as described above is displayed in Figures 2-4 (The curves are obtained from spline fits to the data.). This data has been applied to determine the transport quantities of the present work. 


\section{RESULTS AND DISCUSSION}

The potential data described in the previous section was used as input to our scattering calculation outlined in Section II to calculate the transport collision cross sections. In some cases, it was necessary to extrapolate the short range repulsive region of the potentials to obtain cross sections at high energies. Hence, we have fit either an exponential or a shielded coulomb potential function to the potential data. Consequently, the cross sections at high energies will reflect the uncertainty introduced by this approximation; however, the resultant error in the collision integrals for the temperature range considered in the present work is expected to be small because the cross sections requiring small values of the classical tuming point are used only in the high energy tail of the integration.

By using the proper asymptotic form of the long-range forces, we are able to obtain meaningful values for the collision cross sections at low energy. At the lowest energies, however, there is an uncertainty in the cross section arising from errors in fitting the potential data. On the other hand, the corresponding uncertainty introduced into the collision integral is not expected to be significant, because the contribution to the integral in Eq. (1) from the low energy cross sections is small.

The collision cross sections were computed for each molecular state using Equation (2) and then averaged over all states using the appropriate statistical weights [3] to obtain the mean collision cross sections, $\overline{\mathrm{Q}}_{\mathbf{n}}$. These mean collision cross sections correspond to measured quantities. The mean transport collision integrals $\sigma^{2} \bar{\Omega}_{\mathrm{n}, \mathrm{s}}$ have been calculated from $\overline{\mathrm{Q}}_{\mathrm{n}}$ according to Eq. (1) and are listed in Tables 1-3. As in previous calculations, we find that major contribution to the scattering comes from the higher-lying states. The contribution to $\sigma^{2} \bar{\Omega}_{2,2}$ from the low-lying bound states for which the potential wells have been experimentally measured, is approximately $15 \%, 25 \%$, and $30 \%$ of the total for ground state $\mathrm{O}-\mathrm{O}, \mathrm{N}-\mathrm{N}$, and $\mathrm{N}-\mathrm{O}$ interactions, respectively,

Employing the potential energy curve of Ref. 19 for the $\mathrm{A}^{\prime 5} \Sigma_{\mathrm{g}}^{+}$state of $\mathrm{N}_{2}$ increased the 
collision cross sections for this state by up to $8 \%$ compared with previous work [3] which used a potential calculated for this state by Krauss and Neumann [20]. The values of the viscosity and thermal conductivity collision integrals for $\mathrm{N}-\mathrm{N}$ collisions are presented by Rainwater et al. [43]; they have been determined from experimental data for the singlet and triplet states and $a b$ initio potential curves for the quintet and septet states of $\mathrm{N}_{2}$. Values of the diffusion collision integral $\sigma^{2} \bar{\Omega}_{1,1}$ and the viscosity collision integral $\sigma^{2} \bar{\Omega}_{2,2}$ are listed in Ref. 43 for temperatures in the range $1000 \mathrm{~K}$ to $20,000 \mathrm{~K}$. At the lowest temperature their values are about $8 \%$ higher than the values of the collision integrals calculated from the improved potential data of the present work, but the difference decreases to about $1-3 \%$ at the higher temperature. Values of the diffusion and viscosity integrals for $\mathrm{O}-\mathrm{O}$ collisions have been calculated by Capitelli and Ficocelli [45] using energies from a valence configuration interaction calculation of Schaeffer and Harris [46]. More recently, Holland and Biolsi [47] have calculated these integrals from the results of Ref. 27 for temperatures in the range $2,000 \mathrm{~K}$ to $20,000 \mathrm{~K}$. The values of these transport integrals calculated from the present work differ from the tabulated results of Ref. 45 and Ref. 47 by at most $5 \%$ and $3 \%$ respectively. References to earlier work based on various potential models can be found in Refs. 44,45 and 47.

We have applied our calculated values of the collision integrals to determine transport quantities for nitrogen and oxygen. The viscosity $\eta$, and thermal conductivity $\lambda$ are shown in Figs. 5 and 6 , respectively, for a range of temperatures of interest for the studies of high temperature air in thermodynamic equilibrium. We also show the corresponding quantities for $\mathrm{N}-\mathrm{O}$ interactions (i.e., see dashed lines) for application to gas mixtures. Similarly, the self-diffusion coefficients and the binary diffusion coefficient are displayed in Fig. 7.

We have investigated the second-order contribution to the above transport quantities. We find that the second-order corrections to $\eta$ and $\lambda$ are less than $1 \%$ for the temperature range of Tables 1-3; this is consistent with the corresponding small corrections for scattering by hard spheres [7]. Likewise, the second order corrections to the diffusion coefficients are found to be less than $1 \%$ but, in this case much less than the $8 \%$ correction for scattering by hard spheres. Note that 
the values of $\lambda$ for $\mathrm{N}-\mathrm{N}$ and $\mathrm{O}-\mathrm{O}$ interactions are nearly indistinguishable on this plot. This can be explained by the fact that diffusion cross sections are more sensitive to the behavior of the interaction potentials at large $r$.

We have also considered a gas composed of a mixture of nitrogen and oxygen atoms in thermal equilibrium. The values of $\eta, \lambda$, and the thermal diffusion function $\alpha_{\mathrm{T}}$, the ratio of the thermal diffusion coefficient for oxygen to the binary diffusion coefficient, are shown in Figs. 8-10, respectively, for various molar concentrations of the components ranging from a pure nitrogen gas to a pure oxygen gas. We note that the value of $\lambda$ for the mixture falls below the curve for pure oxygen with increasing concentrations of nitrogen, even though the value of $\lambda$ for pure nitrogen is about the same as that for pure oxygen; on the other hand, we also note that this behavior is consistent with the fact that the dashed curve of Fig. 6 lies considerably below both the oxygen and nitrogen curves. A similar anomalous behavior occurs in the value of $\eta$ for the mixture (see Fig. 8, in this case adding oxygen to a nitrogen enriched gas) with a similar explanation; but, the effect is much less pronounced. We further note that the value of $\alpha_{\mathrm{T}}$ for the mixture is sensitive to the relative concentrations. This behaviour of transport properties with concentration can be readily seen with the aid of Fig. 11. This figure shows the variation of $\eta, \lambda$, and $\alpha_{\mathrm{T}}$ with concentration at $10,000 \mathrm{~K}$. We find that the slope of the curve for $\alpha_{\mathrm{T}}$ is nearly linear for all mixture ratios.

\section{CONCLUDING REMARKS}

We report accurate values of the collision integrals for all the interactions of nitrogen and oxygen atoms in their ground states (Tables 1-3). We have combined accurate ab initio results with experimental data to obtain realistic potential curves. We have applied these potential curves along with the proper asymptotic form of the long-range forces to compute precise values of the collision integrals, covering a broad range of translational temperatures, using our recently developed general, semi-classical scattering code. Our tabulated values of the collision integrals are intended to reduce the uncertainty in future predictions of the transport properties of 
nonequilibrium air, particularly at high translational temperatures. The transport properties of gas mixtures of oxygen and nitrogen atoms have been presented as an illustration of the use of the tabulated collision integrals.

We find that the major contributions to the collision integrals come from the higher-lying states. However, the potential curves of these states are generally not amenable to determination by experiment. Consequently, many previous calculations of transport properties by others are based upon poor representations of these curves. The results of large scale-molecular structure calculations have been essential for determining unknown potentials for our calculations to obtain accurate transport integrals.

In past work, we have reported calculations of the collision integrals of the atoms of nitrogen and oxygen with their corresponding ions [2]. In more recent work, we have extended these results to include collision integrals for $\mathrm{N}-\mathrm{O}^{+}$interactions and plan to incorporate $\mathrm{N}^{+}-\mathrm{O}$ in our tabulations. These additional collision integrals should then allow us to define the transport properties of high temperature air.

\section{REFERENCES}

[1] Partridge, H., Bauschlicher, Jr., C.W., and Stallcop, J.R., "N $\mathrm{N}_{2}^{+}$Bound Quartet and Sextet State Potential Energy Curves", Journal of Quantitative Spectroscopy and Radiative Transfer, Vol. 33, 1985, pp. 653-655.

[2] Levin, E., Partridge, H., and Stallcop, J.R., "High Temperature Transport Properties of Air", AIAA paper 87-1632, June 1987.

[3] Levin, E., Partridge, H., and Stallcop, J.R., "High Temperature Transport Properties of Air: N-O Interaction Energies and Collision Integrals", AIAA paper 88-2660, June 1988.

[4] Parridge, H., and Stallcop, J.R., " $\mathrm{N}^{+}-\mathrm{N}$ and $\mathrm{O}^{+}-\mathrm{O}$ Interaction Energies, Dipole Transition Moments, and Transport Cross Sections", AlAA Progress in Astronautics and Aeronautics: Thermophysical Aspects of Re-entry Flows, Vol. 103, edited by James N. Moss and Carl D. 
Scott, AIAA, New York, 1986, pp. 243-260.

[5] Stallcop, J.R., and Partridge, H., "N $\mathrm{N}^{+}-\mathrm{N}$ Long-range Interaction Energies and Resonance Charge Exchange", Physical Review A, Vol. 32, 1985, pp. 639-642.

[6] Stallcop, J.R., "Semiclassical Elastic Scattering Cross Sections for a Central Field Potential Function", NASA SP-3052, Scientific and Technical Information Division, Washington, D.C., 1969.

[7] Chapman, S., and Cowling, T.G., "The Mathematical Theory of Non-uniform Gases", Cambridge University Press, New York, 1952, 2nd ed.

[8] Hirschfelder, J.O., Curtiss, C.F., and Bird, R.B., "Molecular Theory of Gases and Liquids", John Wiley \& Sons, Inc., New York, 1954.

[9] Maitland, G.C., Rigby, M., Smith, E.B., and Wakeham, W.A., "Intermolecular Forces. Their Origin and Determination", Oxford University Press, 1981.

[10] Stallcop, J. R., unpublished.

[11] Bauschlicher, Jr., C.W., and Langhoff, S.R., "Full CI Benchmark Calculations on $\mathrm{N}_{2}$, NO, and $\mathrm{O}_{2}$ : A Comparison of Methods for Describing Multiple Bonds", Journal of Chemical Physics, Vol. 86, 1987, pp. 5595-5599.

[12] Partridge, H., Bauschlicher, Jr., C.W., Langhoff, S.R., and Taylor, P.A., to be submitted.

[13] Huber, K.P. and Herzberg, G., "Molecular Spectra and Molecular Structure; IV Constants of Diatomic Molecules", Van Nostrand Reinhold, N.Y., 1979.

[14] Zeiss, G.D. and Meath, E.A., "Dispersion Energy Constants $C_{6}(A, B)$, Dipole Oscillator Strength Sums and Refractivities for $\mathrm{Li}, \mathrm{N}, \mathrm{O}, \mathrm{H}_{2}, \mathrm{O}_{2}, \mathrm{NH}_{3}, \mathrm{H}_{2} \mathrm{O}, \mathrm{NO}$, and $\mathrm{N}_{2} \mathrm{O}^{\prime \prime}$, Molecular Physics, Vol. 33, 1977, pp. 1155-1176.

[15] Chang, T.V., "Moderately Long-Range Interatomic Forces", Reviews of Modern Physics, Vol. 39, 1967, pp. 911-942.

[16] Lofthus, A. and Krupenie, P.H., "The Spectrum of Molecular Nitrogen", Journal of Physical and Chemical Reference Data, Vol. 6, 1977. pp. 113-307.

[17] Roux, F., Michaud, F., and Verges, J., "High-Resolution Fourier Spectrometry of the ${ }^{14} \mathrm{~N}_{2}$ Infrared Emission Spectrum: Extensive Analysis of the $\mathrm{B}^{3} \Pi_{\mathrm{g}}-\mathrm{A}^{3} \Sigma_{\mathrm{u}}^{+}$System", Journal of 
Molecular Spectroscopy, Vol. 97, 1983, pp. 253-265.

[18] Ermler, W.C., McLean, A.D., and Mulliken, R.S., "Ab Initio Study of Valence State Potential Energy Curves of $\mathrm{N}_{2}$ ", Journal of Physical Chemistry, Vol. 86, 1982, pp. 1305-1314.

[19] Partridge, H., Langhoff, S.R., Bauschlicher, Jr., C.W., and Schwenke, D.W., "Theoretical Study of the $\mathrm{A}^{\prime 5} \Sigma_{\mathrm{g}}^{+}$and $\mathrm{C}^{\prime \prime} \Pi_{\mathrm{u}}$ states of $\mathrm{N}_{2}$ : Implications for the $\mathrm{N}_{2}$ Afterglow", Journal of Chemical Physics, Vol.88, 1988, pp. 3174-3186.

[20] Krauss, M. and Neumann, D.B., "The ${ }^{5} \Sigma_{\mathrm{g}}^{+}$States of $\mathrm{N}_{2}$ ", Molecular Physics, Vol. 32, 1976, pp. 101-112.

[21] Huber, K.P. and Vervloet, M., "Rotational Analysis of the Herman Infrared Bands of Nitrogen", Journal of Chemical Physics, Vol. 89, 1988, pp. 5957-5959.

[22] Dunning, T.H., Jr., Cartwright, D.C., Hunt, W.J., Hay, P.J. and Bobrowicz, F.W., "Generalized Valence Bond Calculations on the Ground State $\left(\mathrm{X}^{1} \Sigma_{\mathrm{g}}^{+}\right)$of Nitrogen", Journal of Chemical Physics, Vol. 64, 1976, pp. 4755-4766.

[23] Ermler, W.C., Clark, J.P., and Mulliken, R.S., "Ab initio Calculations of Potential Energy Curves and Transition Moments of ${ }^{1} \Sigma_{\mathrm{g}}^{+}$and ${ }^{1} \Sigma_{\mathrm{u}}^{+}$of $\mathrm{N}_{2}$ ", Journal of Chemical Physics, Vol. 86, 1987, pp. 370-375.

[24] Partridge, H., Langhoff, S.R., and Bauschlicher, Jr., C.W., "Theoretical Study of the ${ }^{7} \Sigma_{u}^{+}$ State of $\mathrm{N}_{2}{ }^{\prime \prime}$, Journal of Chemical Physics, Vol. 84, 1986, pp. 6901-6906.

[25] Ferrante, R.F. and Stwalley, W.C., "Spin-Polarized Atomic Nitrogen and the ${ }^{7} \Sigma_{u}^{+}$State of $\mathrm{N}_{2}{ }^{\prime}$, Journal of Chemical Physics, Vol. 84, 1983, pp. 3107-3111.

[26] Partridge, H., Langhoff, S.R., and Bauschlicher, Jr., C.W., to be submitted.

[27] Saxon, R.P. and Liu, B., "Ab Initio Configuration Interaction Study of the Valence States of $\mathrm{O}_{2}{ }^{2}$, Journal of Chemical Physics, Vol. 67, 1977, pp. 5432-5441.

[28] Krupenie, P.H., "The Spectrum of Molecular Oxygen", Journal of Physical and Chemical Reference Data, Vol. 1, 1972, pp. 423-534.

[29] Ramsay, D.A., "High-Resolution Studies of the Near-Ultraviolet Bands of Oxygen: I: the $c^{1} \Sigma_{\mathrm{u}}^{-}-\mathrm{X}^{3} \Sigma_{\mathrm{g}}^{-}$System", Canadian Journal of Physics, Vol. 64, 1986, pp. 717-720.

[30] Borrell, P.M., Borrell, P., and Ramsay, D.A., "High-Resolution Studies of the Near- 
Ultraviolet Bands of Oxygen: II: the $\mathrm{A}^{3} \Sigma_{\mathrm{u}}^{+}-\mathrm{X}^{3} \Sigma_{\mathrm{g}}^{-}$System", Canadian Journal of Physics, Vol. 64, 1986, pp. 721-725.

[31] Coquart, B. and Ramsay, D.A., "High-Resolution Studies of the Near-Ultraviolet Bands of Oxygen: III: the $\mathrm{A}^{\prime 3} \Delta_{\mathrm{u}}-\mathrm{X}^{3} \Sigma_{\mathrm{g}}^{-}$System", Canadian Journal of Physics, Vol. 64, 1986, pp. 726732.

[32] Slanger, T.G. and Cosby, P.C., "O $\mathrm{O}_{2}$ Spectroscopy Below $5.1 \mathrm{eV",} \mathrm{Journal} \mathrm{of} \mathrm{Physical}$ Chemistry, Vol. 92, 1988, pp. 267-285.

[33] Nieh, J. and Valentini, J.J., "Molecular Potentials from CARS Photofragment Spectroscopy: Spectroscopic Constants and Potential Energy Curve for $\mathrm{O}_{2}\left(\mathrm{a}^{1} \Delta_{\mathrm{g}}\right)$ ", Journal of Physical Chemistry, Vol. 91, 1987, pp. 1370-1374.

[34] Buenker, R.J. and Peyerimhoff, S.D., "Ab Initio Study of the Mixing of Valence and Rydberg States in $\mathrm{O}_{2}$ : CI Potential Curves for the ${ }^{3} \Sigma_{\mathrm{u}}^{-},{ }^{3} \Delta_{\mathrm{u}}$, and ${ }^{3} \Pi_{\mathrm{u}}$ States", Chemical Physics Letters, Vol. 34, 1975, pp. 225-231.

[35] Guberman, S.L. and Dalgarno, A., "Absorption Into the ${ }^{3} \Pi_{\mathrm{u}}$ State of Molecular Oxygen", Journal of Geophysical Research, Vol. 84, 1979, pp. 4437-4440.

[36] Saxon, R.P. and Liu, B., "Ab Initio Configuration Interaction Study of the Rydberg States of $\mathrm{O}_{2}$. I. A General Computational Procedure for Diabatic Molecular Rydberg States and Test Calculations on the ${ }^{3} \Pi_{\mathrm{g}}$ States of $\mathrm{O}_{2}{ }^{\prime}$, Journal of Chemical Physics, Vol. 73, 1980, pp. 870-875. [37] Saxon, R.P. and Liu, B., "Ab Initio Study of the Rydberg States of $\mathrm{O}_{2}$. II. Calculations on the ${ }^{3} \Sigma_{\mathrm{g}}^{-},{ }^{3} \Sigma_{\mathrm{u}}^{-},{ }^{3} \Pi_{\mathrm{g}},{ }^{1} \Pi_{\mathrm{g}}$, and ${ }^{1} \Sigma_{\mathrm{g}}^{+}$Symmetries", Journal of Chemical Physics, Vol. 73, 1980, pp. 876-880.

[38] Cartwright, D.C., Hunt, W.J., Williams, W., Trajmar, S., and Goddard, III, W.A., "Theoretical and Experimental (Electron-Impact) Studies of the Low-Lying Rydberg States in $\mathrm{O}_{2}$ ", Physical Review A., Vol. 8, 1973, pp. 2436-2448.

[39] Cartwright, D.C., Fiamengo, N.A., Williams, W. and Trajmar, W., "Decomposition of the Photoabsorption Schumann-Runge Continuum in $\mathrm{O}_{2}$ ", Journal of Physics B., Vol. 9, 1976, p. L419.

[40] Trajmar, S., Cartwright, D.C. and Hall, R.I., "Electron Impact Excitation of the Rydberg 
States in $\mathrm{O}_{2}$ in the 7-10 eV Energy Loss Region", Journal of Chemical Physics, Vol. 65, 1976, pp. 5275-5279.

[41] Langhoff, S.R., Bauschlicher, Jr., C.W., and Partridge, H., "Theoretical Study of the NO $\gamma$ System", Journal of Chemical Physics, Vol. 89, 1988, pp. 4909-4913.

[42] Partridge, H., Bauschlicher, Jr., C.W., and Langhoff, S.R., "Theoretical Study of the ${ }^{6} \Pi,{ }^{6} \Sigma^{+}$and ${ }^{4} \Sigma^{+}$Van der Waals States of NO", to be submitted.

[43] Grein, F. and Kapur, A., "Low-Lying Valence and Rydberg States of Nitric Oxide NO", Journal of Chemical Physics, Vol. 77, 1982, pp. 415-423.

[44] Rainwater, J.C., Biolsi, L., Biolsi, K.J., and Holland, P.M., "Transport Properties of Ground State Nitrogen Atoms", Journal of Chemical Physics, Vol. 79, 1983, pp. 1462-1465.

[45] Capitelli, M. and Ficocelli V, E., "Collision Integrals of Oxygen Atoms in Different Electronic States", Journal of Physics B., Vol. 5, 1972, pp. 2066-2073.

[46] Schaeffer III, H.F. and Harris, F.E., "Ab Initio Calculations on 62 Low-Lying States of the $\mathrm{O}_{2}$ Molecule", Journal of Chemical Physics, Vol. 48, 1968, pp. 4946-4955.

[47] Holland, P.M. and Biolsi, W., "Transport Properties of Ground State Oxygen Atoms", Journal of Chemical Physics, Vol. 89, 1988, pp. 3203-3210. 


\begin{tabular}{|c|c|c|c|c|c|c|c|c|c|}
\hline \multicolumn{10}{|c|}{ TABLE 1. 0-O TRANSPORT COLLISION INTEGRALS $\left(10^{-16} \mathrm{~cm}^{2}\right)$} \\
\hline $\mathrm{T}(\mathrm{K})$ & $\sigma^{2} \bar{\Omega}_{1,1}$ & $\sigma^{2} \bar{\Omega}_{1,2}$ & $\sigma^{2} \bar{\Omega}_{1,3}$ & $\sigma^{2} \bar{\Omega}_{1.4}$ & $\sigma^{2} \bar{\Omega}_{1,5}$ & $\sigma^{2} \bar{\Omega}_{2,2}$ & $\sigma^{2} \bar{\Omega}_{2,3}$ & $\sigma^{2} \bar{\Omega}_{2,4}$ & $\sigma^{2} \bar{\Omega}_{3,3}$ \\
\hline 250 & 9.035 & 8.091 & 7.461 & 6.991 & 7.287 & 9.953 & 9.242 & 8.727 & 8.698 \\
\hline 300 & 8.534 & 7.649 & 7.053 & 6.602 & 6.569 & 9.459 & 8.799 & 8.308 & 8.237 \\
\hline 500 & 7.281 & 6.528 & 6.014 & 5.580 & 5.164 & 8.217 & 7.636 & 7.118 & 7.084 \\
\hline 750 & 6.426 & 5.773 & 5.326 & 4.929 & 4.574 & 7.332 & 6.792 & 6.288 & 6.302 \\
\hline 1000 & 5.894 & 5.310 & 4.912 & 4.576 & 4.311 & 6.758 & 6.260 & 5.825 & 5.818 \\
\hline 2000 & 4.837 & 4.393 & 4.093 & 3.866 & 3.688 & 5.584 & 5.207 & 4.926 & 4.844 \\
\hline 3000 & 4.331 & 3.939 & 3.667 & 3.457 & 3.285 & 5.026 & 4.702 & 4.461 & 4.356 \\
\hline 4000 & 4.003 & 3.634 & 3.372 & 3.167 & 2.999 & 4.669 & 4.369 & 4.139 & 4.031 \\
\hline 5000 & 3.760 & 3.403 & 3.146 & 2.946 & 2.781 & 4.407 & 4.117 & 3.892 & 3.786 \\
\hline 6000 & 3.567 & 3.218 & 2.965 & 2.768 & 2.606 & 4.198 & 3.914 & 3.694 & 3.589 \\
\hline 7000 & 3.407 & 3.063 & 2.815 & 2.620 & 2.460 & 4.025 & 3.746 & 3.529 & 3.425 \\
\hline 8000 & 3.270 & 2.931 & 2.686 & 2.494 & 2.336 & 3.877 & 3.603 & 3.389 & 3.285 \\
\hline 9000 & 3.151 & 2.816 & 2.574 & 2.384 & 2.229 & 3.749 & 3.478 & 3.266 & 3.163 \\
\hline 10000 & 3.046 & 2.715 & 2.474 & 2.288 & 2.136 & 3.635 & 3.367 & 3.157 & 3.055 \\
\hline 11000 & 2.952 & 2.624 & 2.386 & 2.202 & 2.053 & 3.533 & 3.267 & 3.059 & 2.959 \\
\hline 12000 & 2.866 & 2.541 & 2.307 & 2.126 & 1.980 & 3.441 & 3.177 & 2.969 & 2.872 \\
\hline 13000 & 2.789 & 2.467 & 2.235 & 2.057 & 1.915 & 3.357 & 3.094 & 2.888 & 2.794 \\
\hline 14000 & 2.717 & 2.399 & 2.170 & 1.995 & 1.856 & 3.279 & 3.018 & 2.812 & 2.722 \\
\hline 15000 & 2.652 & 2.336 & 2.110 & 1.938 & 1.803 & 3.207 & 2.947 & 2.743 & 2.655 \\
\hline 16000 & 2.591 & 2.278 & 2.055 & 1.887 & 1.754 & 3.140 & 2.882 & 2.678 & 2.594 \\
\hline 17000 & 2.534 & 2.224 & 2.005 & 1.839 & 1.709 & 3.078 & 2.820 & 2.618 & 2.537 \\
\hline 18000 & 2.481 & 2.174 & 1.958 & 1.795 & 1.668 & 3.019 & 2.763 & 2.563 & 2.484 \\
\hline 19000 & 2.432 & 2.128 & 1.914 & 1.754 & 1.629 & 2.964 & 2.709 & 2.511 & 2.434 \\
\hline 20000 & 2.385 & 2.084 & 1.874 & 1.716 & 1.593 & 2.912 & 2.659 & 2.462 & 2.388 \\
\hline 21000 & 2.341 & 2.044 & 1.836 & 1.681 & 1.560 & 2.863 & 2.611 & 2.416 & 2.344 \\
\hline 22000 & 2.300 & 2.005 & 1.800 & 1.647 & 1.528 & 2.816 & 2.566 & 2.373 & 2.303 \\
\hline 23000 & 2.261 & 1.969 & 1.766 & 1.616 & 1.498 & 2.772 & 2.523 & 2.333 & 2.264 \\
\hline 24000 & 2.224 & 1.935 & 1.735 & 1.586 & 1.469 & 2.729 & 2.483 & 2.295 & 2.226 \\
\hline 25000 & 2.189 & 1.902 & 1.704 & 1.558 & 1.442 & 2.689 & 2.445 & 2.259 & 2.191 \\
\hline 30000 & 2.035 & 1.762 & 1.574 & 1.434 & 1.323 & 2.515 & 2.280 & 2.104 & 2.038 \\
\hline 35000 & 1.911 & 1.648 & 1.468 & 1.332 & 1.223 & 2.373 & 2.148 & 1.982 & 1.913 \\
\hline 40000 & 1.808 & 1.554 & 1.378 & 1.246 & 1.137 & 2.255 & 2.040 & 1.879 & 1.809 \\
\hline 45000 & 1.719 & 1.472 & 1.301 & 1.170 & 1.061 & 2.156 & 1.947 & 1.791 & 1.720 \\
\hline 50000 & 1.642 & 1.400 & 1.232 & 1.101 & 0.993 & 2.068 & 1.866 & 1.713 & 1.642 \\
\hline 60000 & 1.513 & 1.279 & 1.114 & 0.984 & 0.878 & 1.924 & 1.729 & 1.578 & 1.511 \\
\hline 70000 & 1.406 & 1.178 & 1.014 & 0.886 & 0.781 & 1.805 & 1.612 & 1.462 & 1.403 \\
\hline 80000 & 1.315 & 1.091 & 0.929 & 0.803 & 0.700 & 1.702 & 1.511 & 1.358 & 1.310 \\
\hline 90000 & 1.236 & 1.015 & 0.855 & 0.731 & 0.629 & 1.612 & 1.421 & 1.266 & 1.229 \\
\hline 100000 & 1.167 & 0.948 & 0.790 & 0.667 & 0.568 & 1.531 & 1.338 & 1.181 & 1.157 \\
\hline
\end{tabular}




\begin{tabular}{|c|c|c|c|c|c|c|c|c|c|}
\hline \multicolumn{10}{|c|}{ TABLE 2. N-N TRANSPORT COLLISION INTEGRALS $\left(10^{-16} \mathrm{~cm}^{2}\right)$} \\
\hline $\mathrm{T}(\mathrm{K})$ & $\sigma^{2} \bar{\Omega}_{1,1}$ & $\sigma^{2} \bar{\Omega}_{1,2}$ & $\sigma^{2} \bar{\Omega}_{1,3}$ & $\sigma^{2} \bar{\Omega}_{1,4}$ & $\sigma^{2} \bar{\Omega}_{1,5}$ & $\sigma^{2} \bar{\Omega}_{2,2}$ & $\sigma^{2} \bar{\Omega}_{2,3}$ & $\sigma^{2} \bar{\Omega}_{2,4}$ & $\sigma^{2} \bar{\Omega}_{3,3}$ \\
\hline 250 & 8.493 & 7.693 & 7.160 & $6 . \overline{766}$ & 7.109 & 9.575 & 8.907 & 8.413 & 8.359 \\
\hline 300 & 8.068 & 7.322 & 6.828 & 6.466 & 6.500 & 9.110 & 8.481 & 8.002 & 7.969 \\
\hline 500 & 7.033 & 6.433 & 6.046 & 5.715 & 5.354 & 7.941 & 7.402 & 6.906 & 7.007 \\
\hline 750 & 6.365 & 5.874 & 5.553 & 5.241 & 4.918 & 7.177 & 6.711 & 6.245 & 6.388 \\
\hline 1000 & 5.962 & 5.535 & 5.243 & 4.965 & 4.718 & 6.723 & 6.312 & 5.933 & 6.023 \\
\hline 2000 & 5.145 & 4.786 & 4.514 & 4.283 & 4.092 & 5.818 & 5.504 & 5.252 & 5.235 \\
\hline 3000 & 4.704 & 4.343 & 4.063 & 3.834 & 3.643 & 5.328 & 5.020 & 4.769 & 4.761 \\
\hline 4000 & 4.388 & 4.020 & 3.738 & 3.511 & 3.323 & 4.975 & 4.665 & 4.416 & 4.412 \\
\hline 5000 & 4.140 & 3.768 & 3.487 & 3.262 & 3.077 & 4.700 & 4.393 & 4.151 & 4.140 \\
\hline 6000 & 3.936 & 3.564 & 3.283 & 3.060 & 2.875 & 4.478 & 4.177 & 3.944 & 3.918 \\
\hline 7000 & 3.764 & 3.391 & 3.112 & 2.891 & 2.707 & 4.295 & 4.000 & 3.771 & 3.733 \\
\hline 8000 & 3.614 & 3.243 & 2.965 & 2.745 & 2.564 & 4.139 & 3.849 & 3.623 & 3.576 \\
\hline 9000 & 3.483 & 3.112 & 2.836 & 2.619 & 2.442 & 4.003 & 3.716 & 3.491 & 3.439 \\
\hline 10000 & 3.366 & 2.996 & 2.723 & 2.509 & 2.335 & 3.883 & 3.598 & 3.373 & 3.318 \\
\hline 11000 & 3.261 & 2.893 & 2.622 & 2.411 & 2.241 & 3.775 & 3.491 & 3.266 & 3.211 \\
\hline 12000 & 3.165 & 2.799 & 2.531 & 2.323 & 2.157 & 3.676 & 3.393 & 3.168 & 3.114 \\
\hline 13000 & 3.077 & 2.713 & 2.448 & 2.245 & 2.082 & 3.586 & 3.304 & 3.079 & 3.025 \\
\hline 14000 & 2.996 & 2.635 & 2.374 & 2.173 & 2.014 & 3.502 & 3.221 & 2.998 & 2.944 \\
\hline 15000 & 2.922 & 2.563 & 2.305 & 2.108 & 1.952 & 3.425 & 3.144 & 2.922 & 2.869 \\
\hline 16000 & 2.853 & 2.497 & 2.242 & 2.049 & 1.896 & 3.353 & 3.073 & 2.852 & 2.800 \\
\hline 17000 & 2.788 & 2.436 & 2.184 & 1.994 & 1.844 & 3.285 & 3.007 & 2.788 & 2.736 \\
\hline 18000 & 2.728 & 2.379 & 2.130 & 1.943 & 1.796 & 3.221 & 2.944 & 2.728 & 2.675 \\
\hline 19000 & 2.672 & 2.325 & 2.080 & 1.896 & 1.751 & 3.162 & 2.886 & 2.671 & 2.619 \\
\hline 20000 & 2.619 & 2.275 & 2.033 & 1.852 & 1.709 & 3.105 & 2.831 & 2.619 & 2.566 \\
\hline 21000 & 2.569 & 2.228 & 1.989 & 1.811 & 1.670 & 3.052 & 2.780 & 2.569 & 2.517 \\
\hline 22000 & 2.521 & 2.184 & 1.948 & 1.772 & 1.634 & 3.002 & 2.731 & 2.523 & 2.470 \\
\hline 23000 & 2.476 & 2.142 & 1.909 & 1.736 & 1.600 & 2.954 & 2.685 & 2.479 & 2.426 \\
\hline 24000 & 2.434 & 2.103 & 1.873 & 1.701 & 1.567 & 2.908 & 2.641 & 2.437 & 2.384 \\
\hline 25000 & 2.394 & 2.065 & 1.838 & 1.669 & 1.537 & 2.865 & 2.600 & 2.397 & 2.344 \\
\hline 30000 & 2.218 & 1.904 & 1.689 & 1.530 & 1.405 & 2.675 & 2.420 & 2.226 & 2.172 \\
\hline 35000 & 2.076 & 1.774 & 1.569 & 1.418 & 1.301 & 2.520 & 2.273 & 2.087 & 2.034 \\
\hline 40000 & 1.957 & 1.667 & 1.472 & 1.328 & 1.217 & 2.390 & 2.151 & 1.970 & 1.920 \\
\hline 45000 & 1.856 & 1.577 & 1.389 & 1.252 & 1.147 & 2.279 & 2.046 & 1.871 & 1.823 \\
\hline 50000 & 1.769 & 1.500 & 1.319 & 1.187 & 1.086 & 2.183 & 1.955 & 1.783 & 1.741 \\
\hline 60000 & 1.626 & 1.372 & 1.203 & 1.082 & 0.989 & 2.020 & 1.801 & 1.638 & 1.603 \\
\hline 70000 & 1.511 & 1.272 & 1.113 & 0.999 & 0.912 & 1.888 & 1.678 & 1.520 & 1.492 \\
\hline 80000 & 1.417 & 1.189 & 1.039 & 0.931 & 0.848 & 1.777 & 1.574 & 1.421 & 1.401 \\
\hline 90000 & 1.339 & 1.119 & 0.976 & 0.873 & 0.792 & 1.683 & 1.484 & 1.335 & 1.322 \\
\hline 100000 & 1.271 & 1.060 & 0.923 & 0.822 & 0.743 & 1.600 & 1.407 & 1.260 & 1.253 \\
\hline
\end{tabular}




\begin{tabular}{|c|c|c|c|c|c|c|c|c|c|}
\hline \multicolumn{10}{|c|}{ TABLE 3. N-O TRANSPORT COLLISION INTEGRALS $\left(10^{-16} \mathrm{~cm}^{2}\right)$} \\
\hline $\mathrm{T}(\mathrm{K})$ & $\sigma^{2} \bar{\Omega}_{1,1}$ & $\sigma^{2} \bar{\Omega}_{1,2}$ & $\sigma^{2} \bar{\Omega}_{1,3}$ & $\sigma^{2} \bar{\Omega}_{1,4}$ & $\sigma^{2} \bar{\Omega}_{1,5}$ & $\sigma^{2} \bar{\Omega}_{2,2}$ & $\sigma^{2} \bar{\Omega}_{2,3}$ & $\sigma^{2} \bar{\Omega}_{2,4}$ & $\sigma^{2} \bar{\Omega}_{3,3}$ \\
\hline 250 & 8.718 & 7.962 & 7.466 & 7.103 & 7.500 & 9.454 & 8.912 & 8.520 & 8.521 \\
\hline 300 & 8.321 & 7.619 & 7.157 & 6.810 & 6.862 & 9.078 & 8.576 & 8.204 & 8.170 \\
\hline 500 & 7.341 & 6.751 & 6.342 & 5.971 & 5.571 & 8.152 & 7.731 & 7.361 & 7.267 \\
\hline 750 & 6.662 & 6.129 & 5.741 & 5.369 & 5.002 & 7.519 & 7.131 & 6.753 & 6.622 \\
\hline 1000 & 6.217 & 5.716 & 5.349 & 5.019 & 4.742 & 7.092 & 6.708 & 6.342 & 6.204 \\
\hline 2000 & 5.257 & 4.834 & 4.541 & 4.317 & 4.143 & 6.061 & 5.691 & 5.400 & 5.315 \\
\hline 3000 & 4.771 & 4.391 & 4.125 & 3.917 & 3.742 & 5.498 & 5.168 & 4.925 & 4.842 \\
\hline 4000 & 4.451 & 4.089 & 3.824 & 3.606 & 3.416 & 5.138 & 4.839 & 4.616 & 4.513 \\
\hline 5000 & 4.210 & 3.849 & 3.576 & 3.347 & 3.149 & 4.879 & 4.593 & 4.371 & 4.257 \\
\hline 6000 & 4.012 & 3.647 & 3.365 & 3.130 & 2.930 & 4.672 & 4.387 & 4.155 & 4.046 \\
\hline 7000 & 3.842 & 3.471 & 3.183 & 2.947 & 2.751 & 4.495 & 4.205 & 3.962 & 3.864 \\
\hline 8000 & 3.692 & 3.317 & 3.026 & 2.792 & 2.600 & 4.338 & 4.040 & 3.790 & 3.705 \\
\hline 9000 & 3.559 & 3.180 & 2.890 & 2.659 & 2.472 & 4.196 & 3.891 & 3.637 & 3.563 \\
\hline 10000 & 3.439 & 3.058 & 2.769 & 2.543 & 2.361 & 4.067 & 3.757 & 3.501 & 3.435 \\
\hline 11000 & 3.330 & 2.948 & 2.662 & 2.440 & 2.263 & 3.948 & 3.635 & 3.380 & 3.320 \\
\hline 12000 & 3.230 & 2.849 & 2.567 & 2.349 & 2.176 & 3.839 & 3.525 & 3.272 & 3.215 \\
\hline 13000 & 3.139 & 2.760 & 2.481 & 2.267 & 2.097 & 3.738 & 3.424 & 3.174 & 3.119 \\
\hline 14000 & 3.055 & 2.677 & 2.402 & 2.193 & 2.027 & 3.645 & 3.332 & 3.085 & 3.030 \\
\hline 15000 & 2.977 & 2.602 & 2.331 & 2.125 & 1.962 & 3.559 & 3.248 & 3.004 & 2.949 \\
\hline 16000 & 2.905 & 2.532 & 2.265 & 2.063 & 1.903 & 3.479 & 3.170 & 2.930 & 2.874 \\
\hline 17000 & 2.837 & 2.468 & 2.204 & 2.005 & 1.849 & 3.404 & 3.098 & 2.861 & 2.804 \\
\hline 18000 & 2.774 & 2.408 & 2.148 & 1.952 & 1.799 & 3.334 & 3.031 & 2.797 & 2.740 \\
\hline 19000 & 2.715 & 2.352 & 2.096 & 1.903 & 1.752 & 3.269 & 2.968 & 2.738 & 2.679 \\
\hline 20000 & 2.659 & 2.300 & 2.047 & 1.857 & 1.708 & 3.208 & 2.909 & 2.682 & 2.623 \\
\hline 21000 & 2.607 & 2.251 & 2.001 & 1.814 & 1.667 & 3.150 & 2.854 & 2.630 & 2.570 \\
\hline 22000 & 2.558 & 2.205 & 1.958 & 1.773 & 1.628 & 3.095 & 2.803 & 2.581 & 2.520 \\
\hline 23000 & 2.511 & 2.161 & 1.917 & 1.735 & 1.592 & 3.043 & 2.754 & 2.534 & 2.472 \\
\hline 24000 & 2.466 & 2.120 & 1.879 & 1.699 & 1.558 & 2.994 & 2.707 & 2.490 & 2.428 \\
\hline 25000 & 2.424 & 2.081 & 1.842 & 1.665 & 1.525 & 2.947 & 2.663 & 2.448 & 2.386 \\
\hline 30000 & 2.240 & 1.911 & 1.685 & 1.517 & 1.385 & 2.744 & 2.472 & 2.266 & 2.203 \\
\hline 35000 & 2.091 & 1.775 & 1.559 & 1.398 & 1.272 & 2.579 & 2.316 & 2.117 & 2.057 \\
\hline 40000 & 1.967 & 1.661 & 1.454 & 1.300 & 1.180 & 2.440 & 2.185 & 1.992 & 1.936 \\
\hline 45000 & 1.860 & 1.566 & 1.365 & 1.217 & 1.101 & 2.322 & 2.073 & 1.884 & 1.832 \\
\hline 50000 & 1.768 & 1.482 & 1.289 & 1.146 & 1.033 & 2.218 & 1.975 & 1.790 & 1.743 \\
\hline 60000 & 1.616 & 1.345 & 1.162 & 1.027 & 0.922 & 2.044 & 1.810 & 1.632 & 1.594 \\
\hline 70000 & 1.493 & 1.234 & 1.060 & 0.932 & 0.833 & 1.903 & 1.675 & 1.502 & 1.474 \\
\hline 80000 & 1.392 & 1.144 & 0.977 & 0.854 & 0.758 & 1.782 & 1.561 & 1.392 & 1.373 \\
\hline 90000 & 1.305 & 1.066 & 0.906 & 0.787 & 0.694 & 1.679 & 1.463 & 1.296 & 1.286 \\
\hline 100000 & 1.231 & 1.000 & 0.844 & 0.729 & 0.638 & 1.588 & 1.375 & 1.210 & 1.210 \\
\hline
\end{tabular}




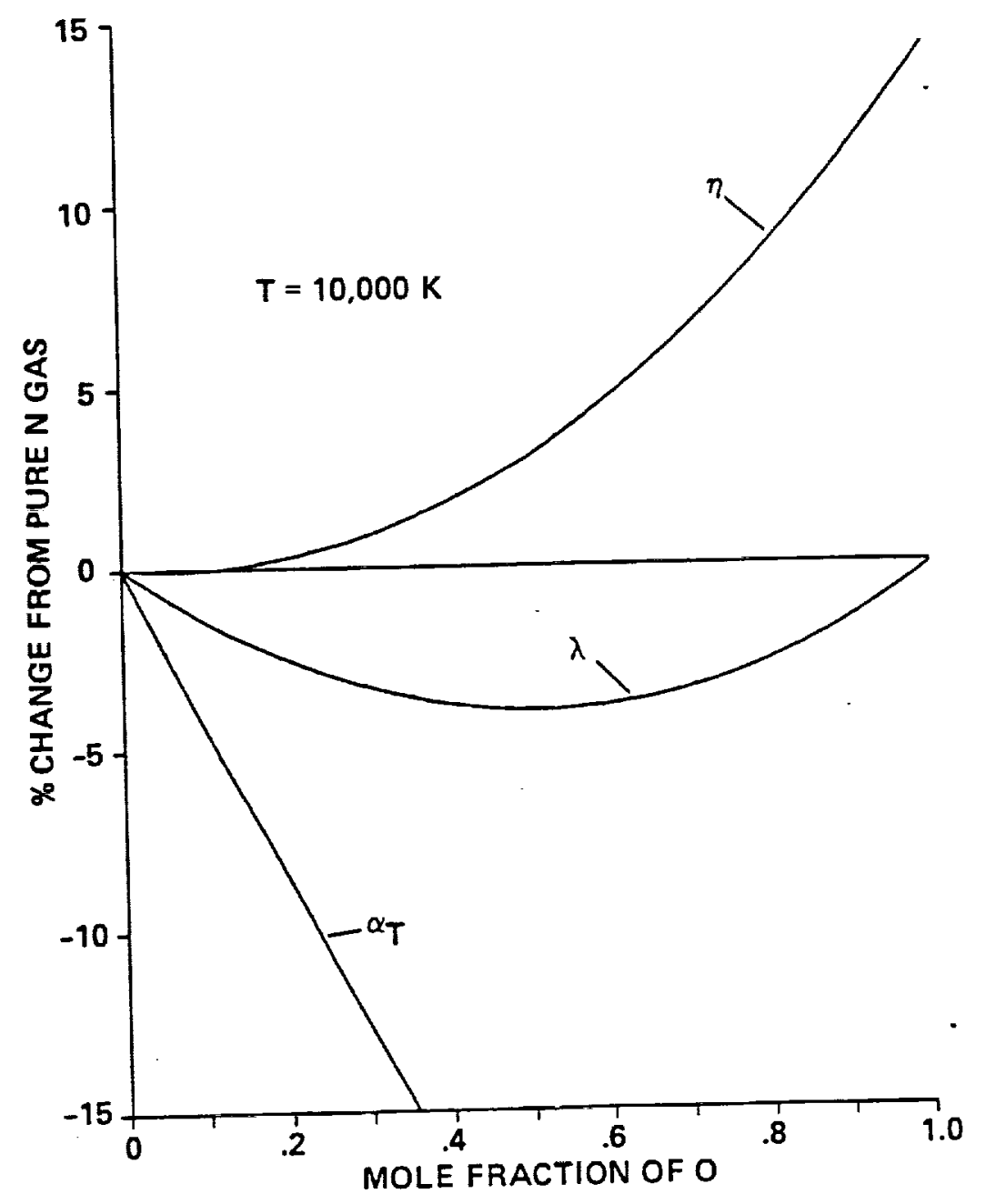

Fig. 11 Variation of transport properties with concentration of oxygen for a mixture of nitrogen and oxygen. 


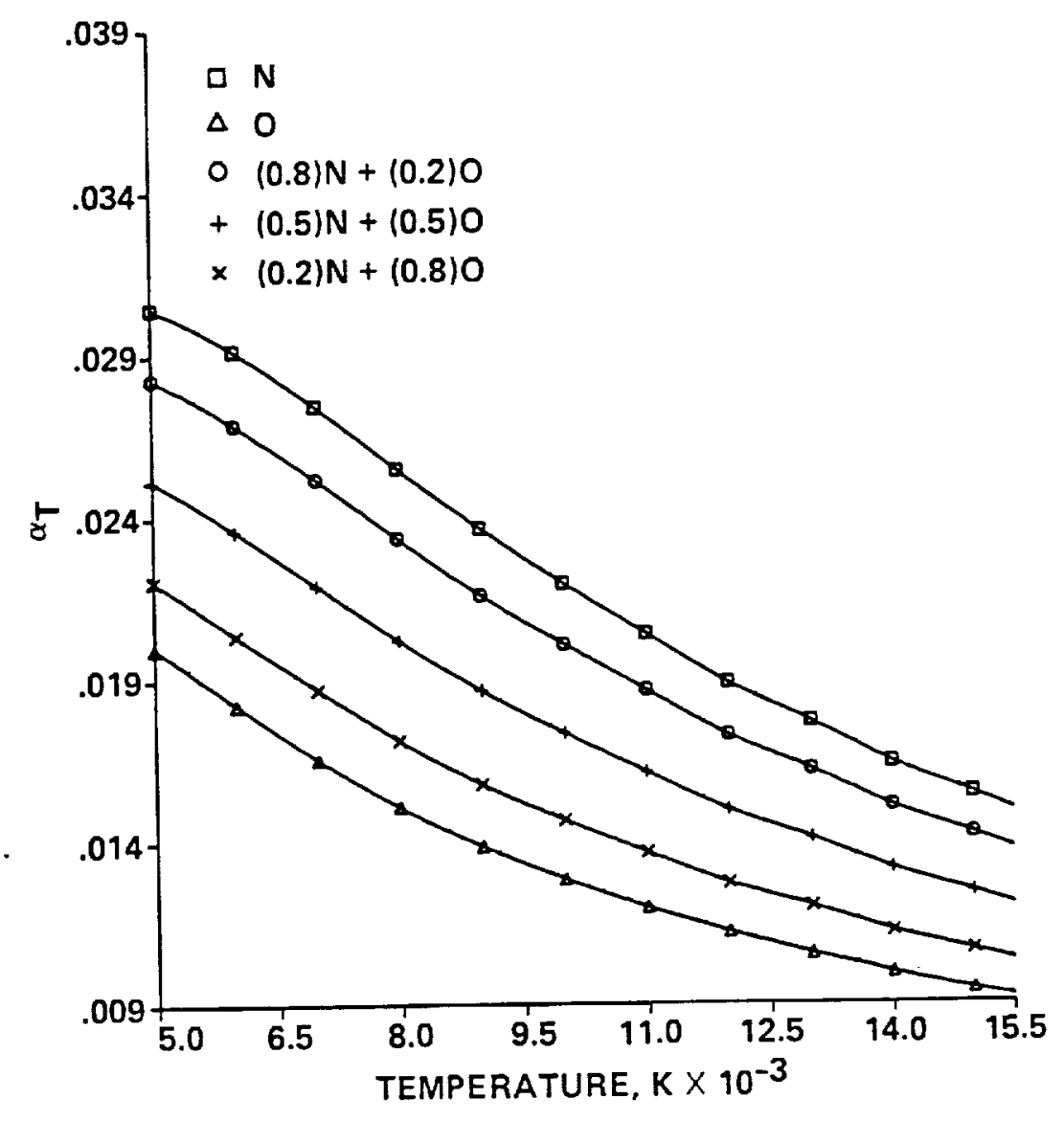

Fig. 10 Thermal diffusion factor for a mixture of nitrogen and oxygen; molar concentrations are specified in parenthesis. 


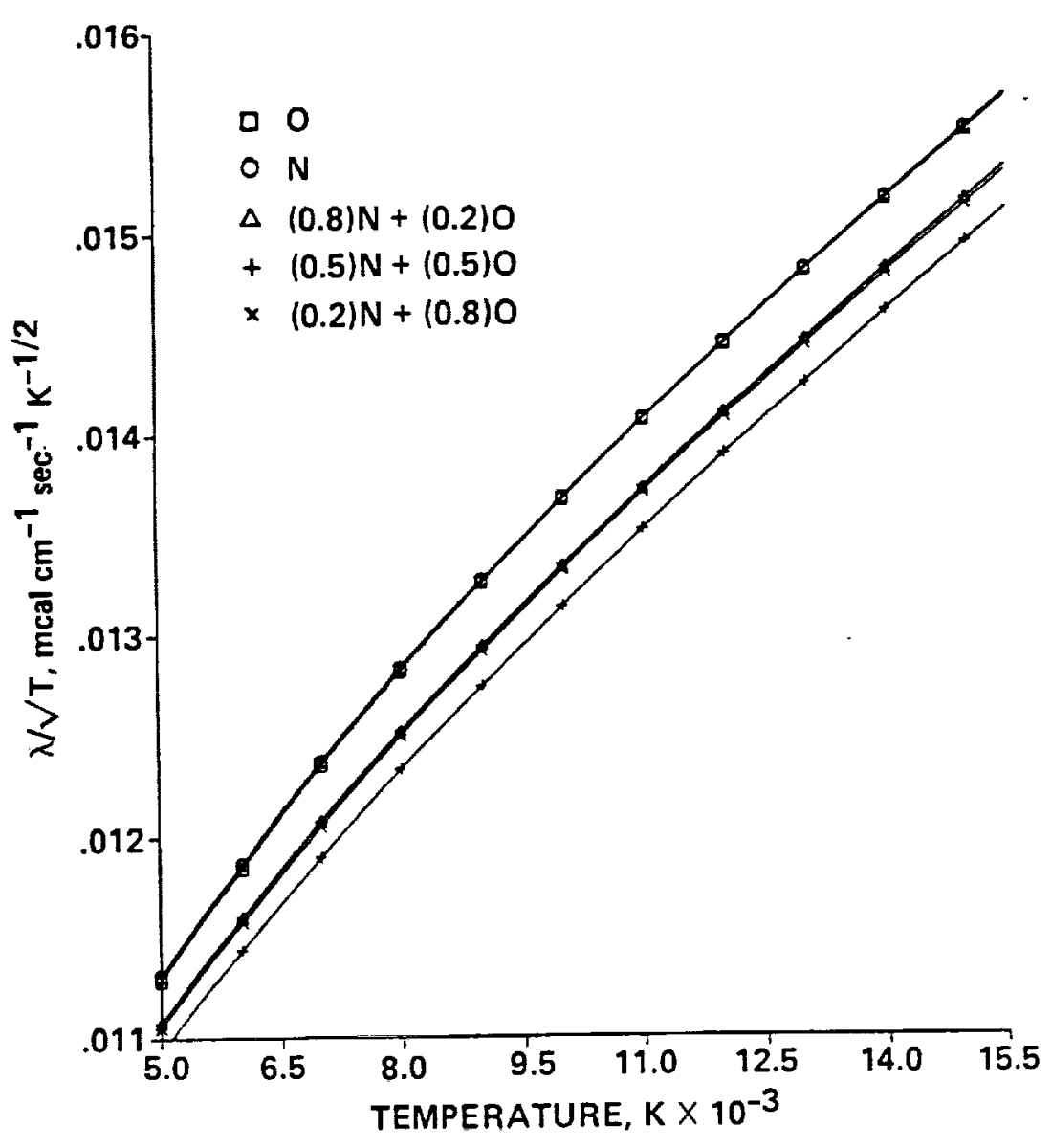

Fig. 9 Thermal conductivity for a mixture of nitrogen and oxygen; molar concentrations are specified in parenthesis. 


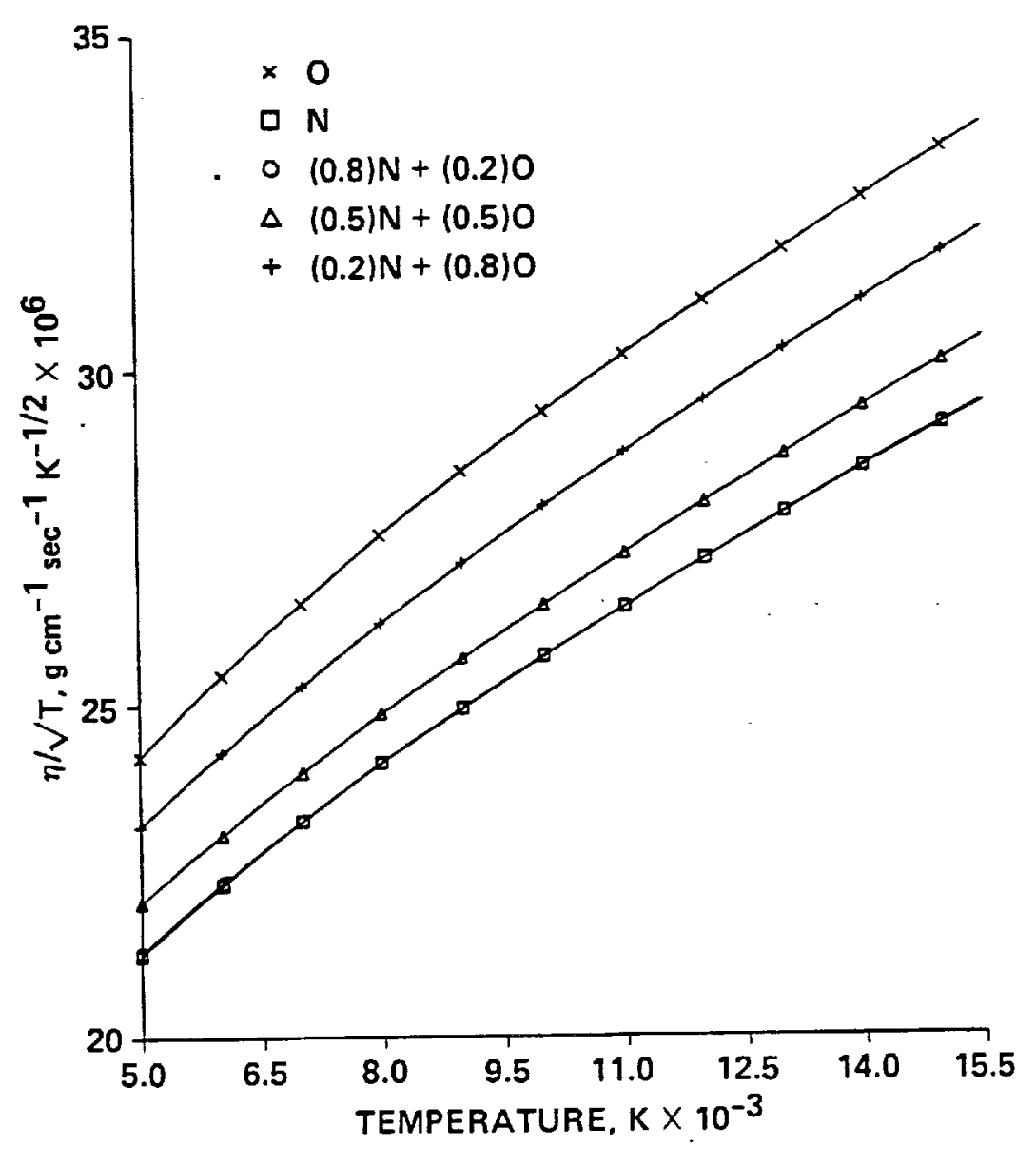

Fig. 8 Viscosity for a mixture of nitrogen and oxygen; molar concentrations are specified in parenthesis. 


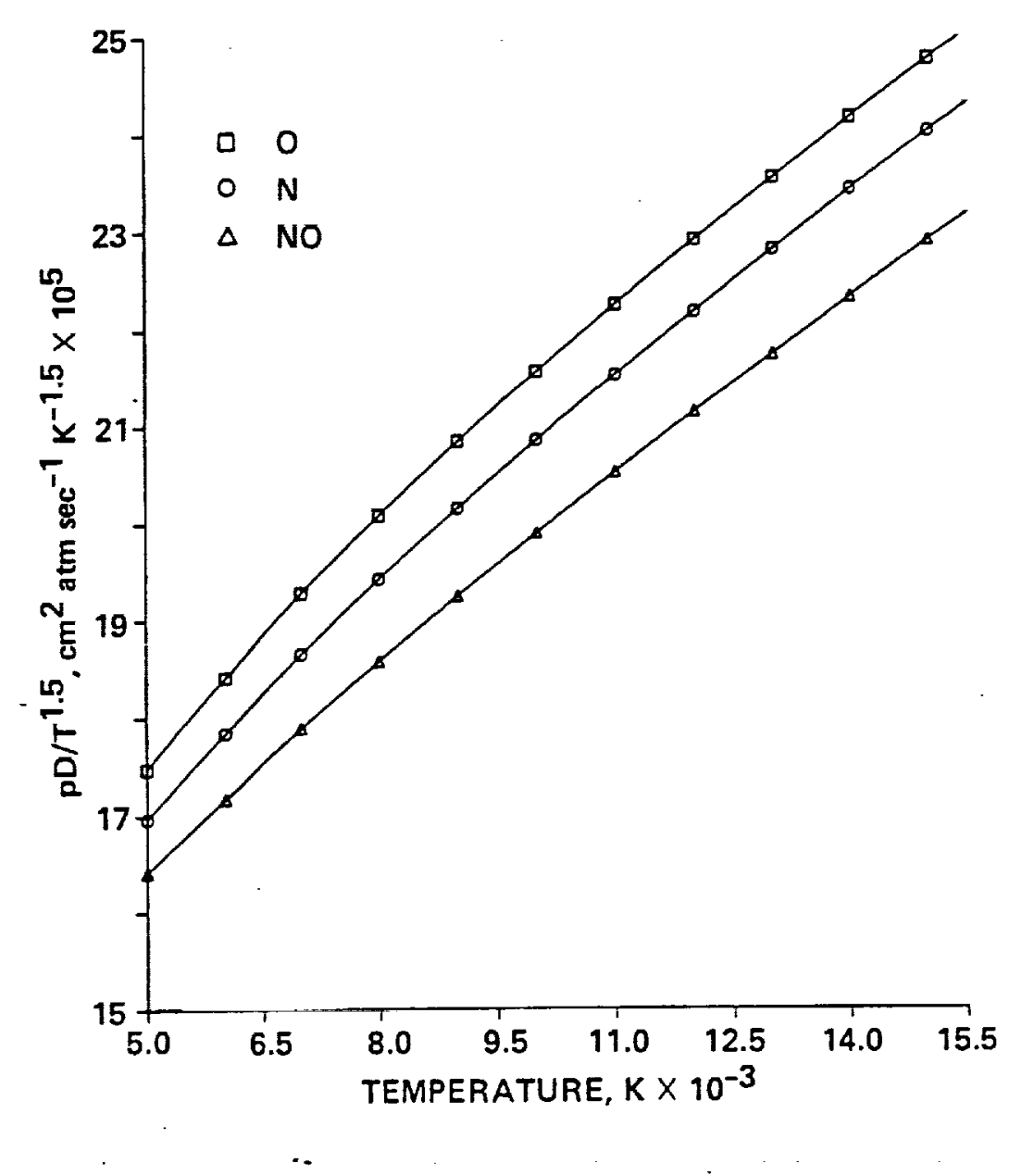

Fig. 7 Self diffusion coefficient for nitrogen and oxygen; binary diffusion coefficient for nitrogen-oxygen ( $p$ is the gas pressure in atmospheres). 


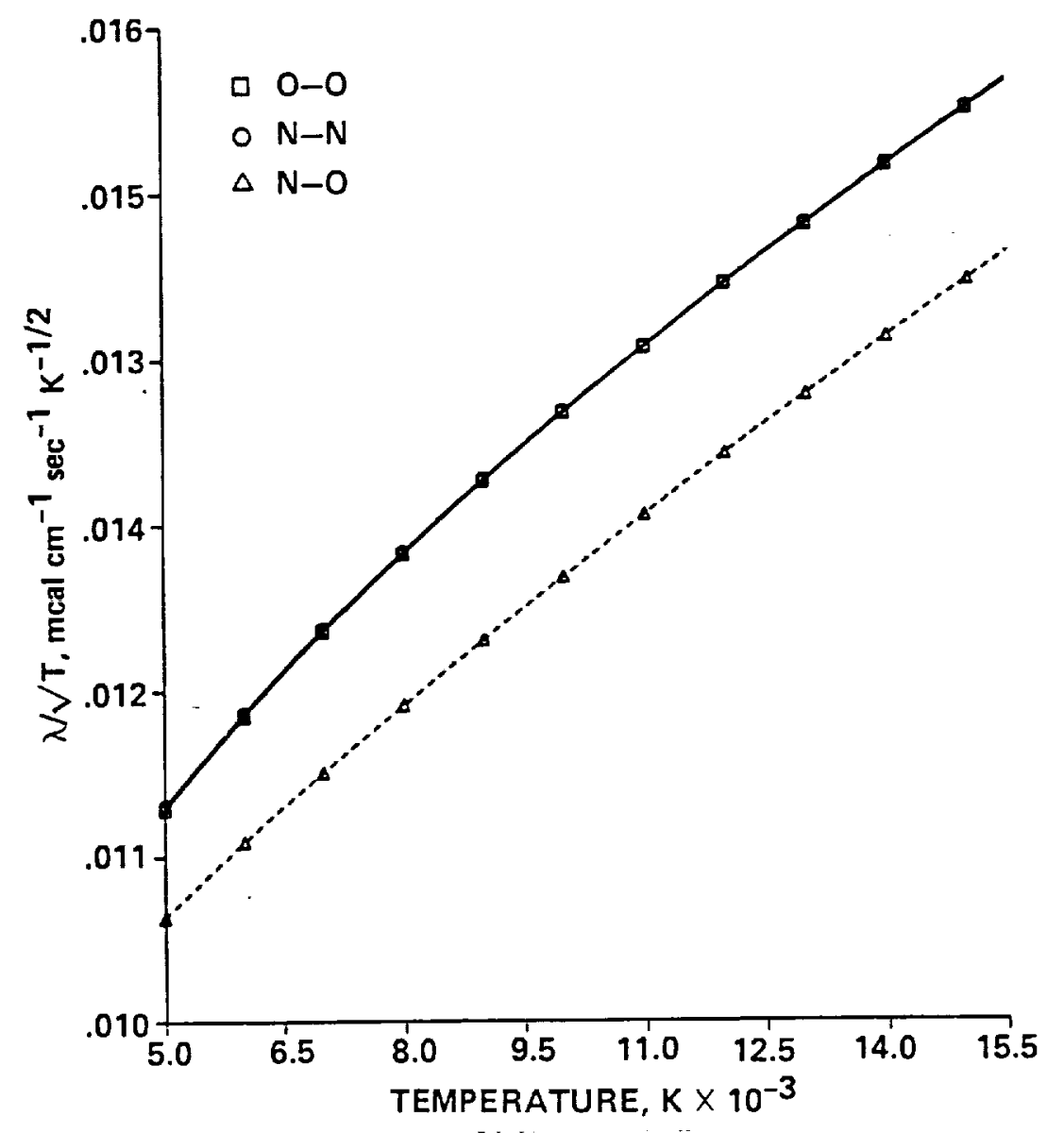

Fig. 6 Thermal conductivity for nitrogen and oxygen; similiar quantity for nitrogen-oxygen interactions (dashed line). 


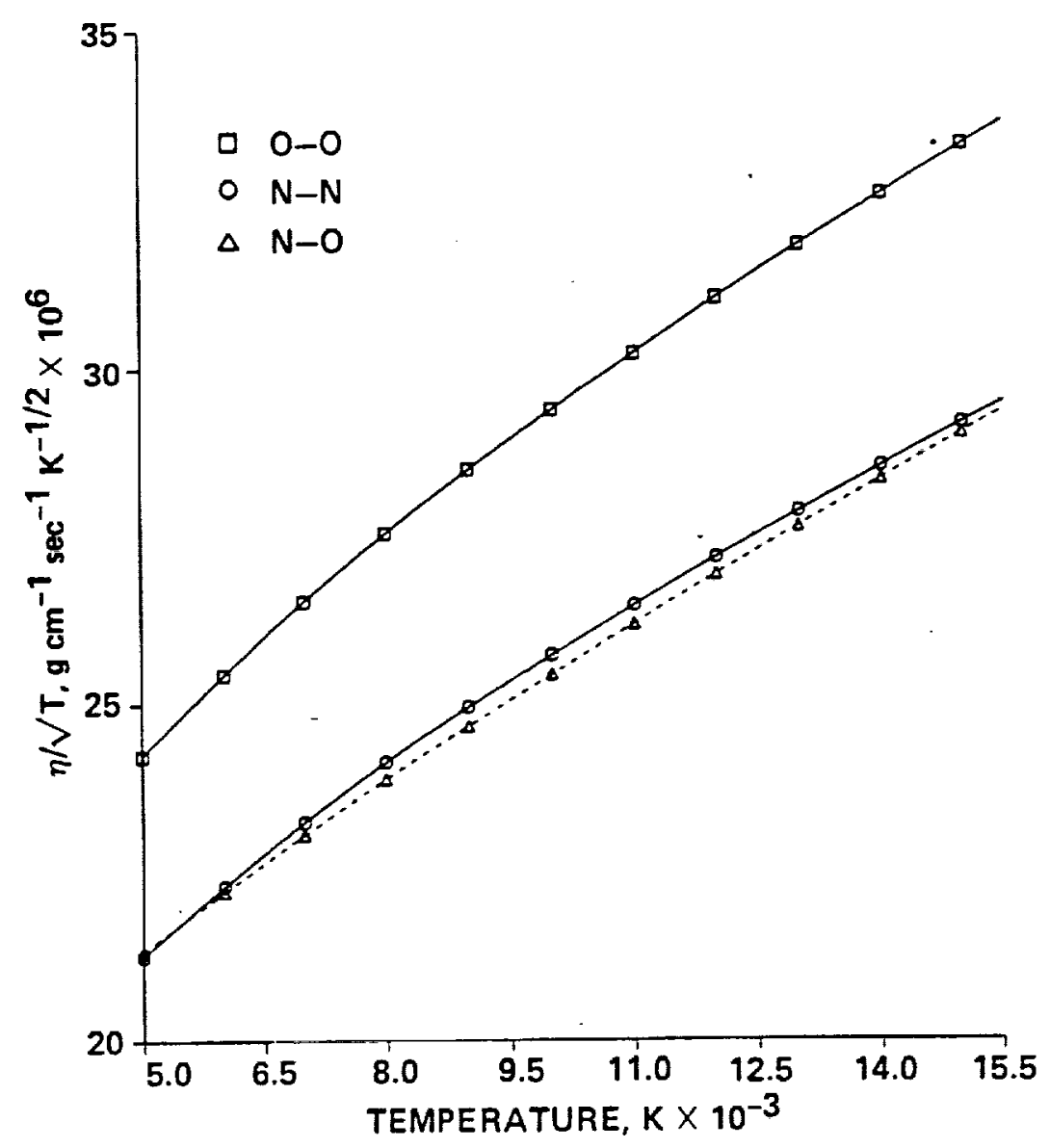

Fig. 5 Viscosity for nitrogen and oxygen; similiar quantity for nitrogen-oxygen interactions (dashed line). 


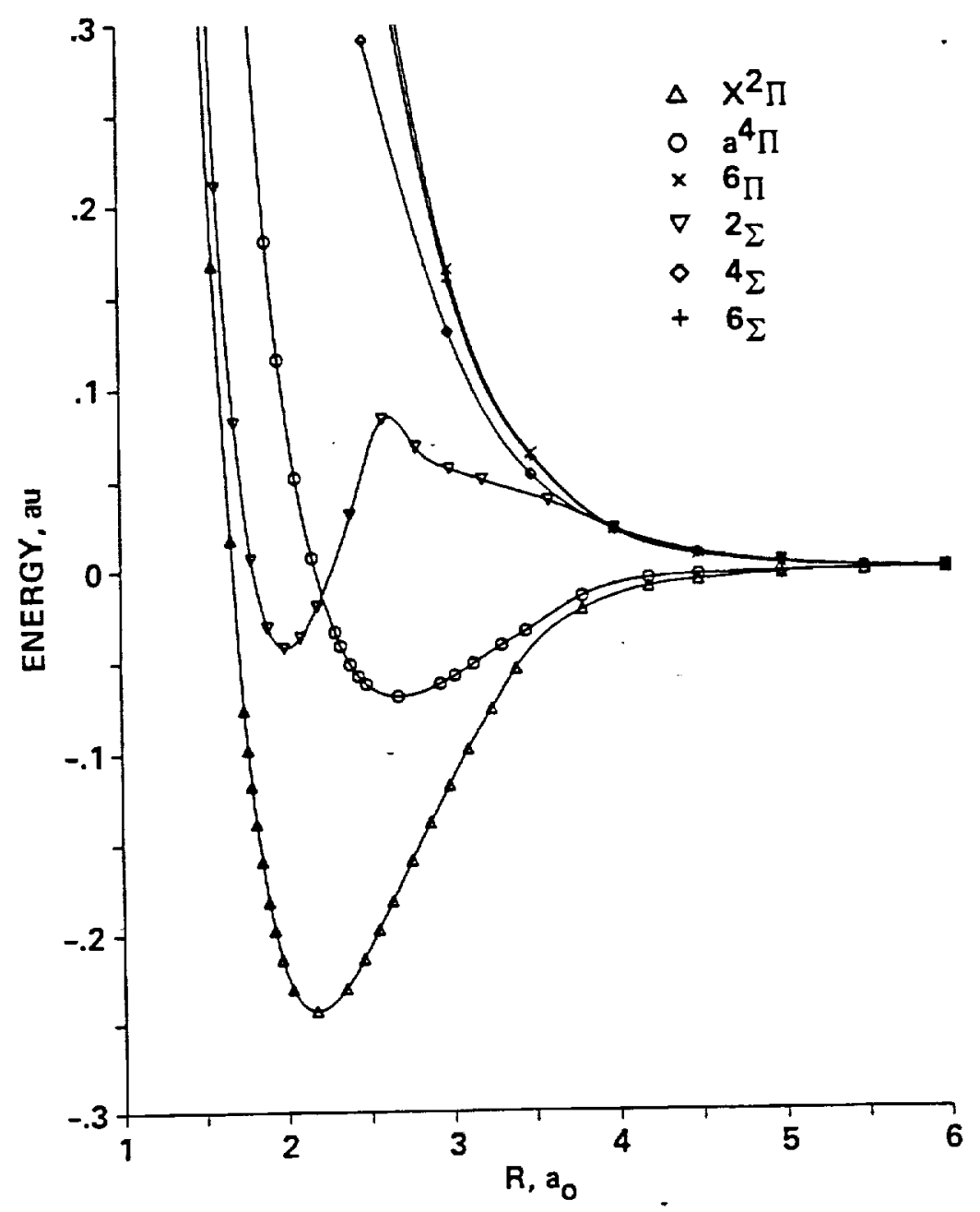

Fig. 4 NO potential energy curves. 


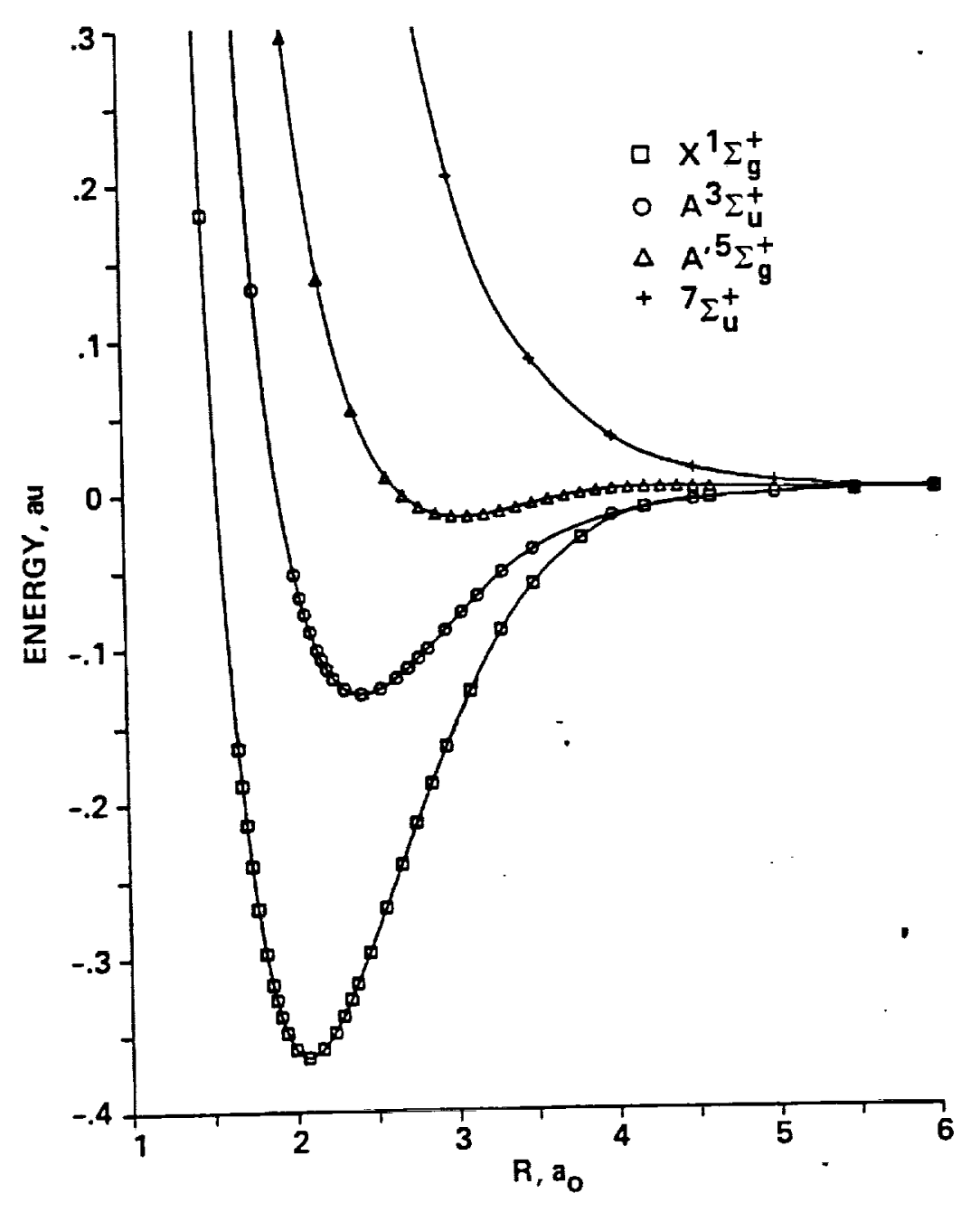

Fig. $3 \mathrm{~N}_{2}$ potential energy curves. 


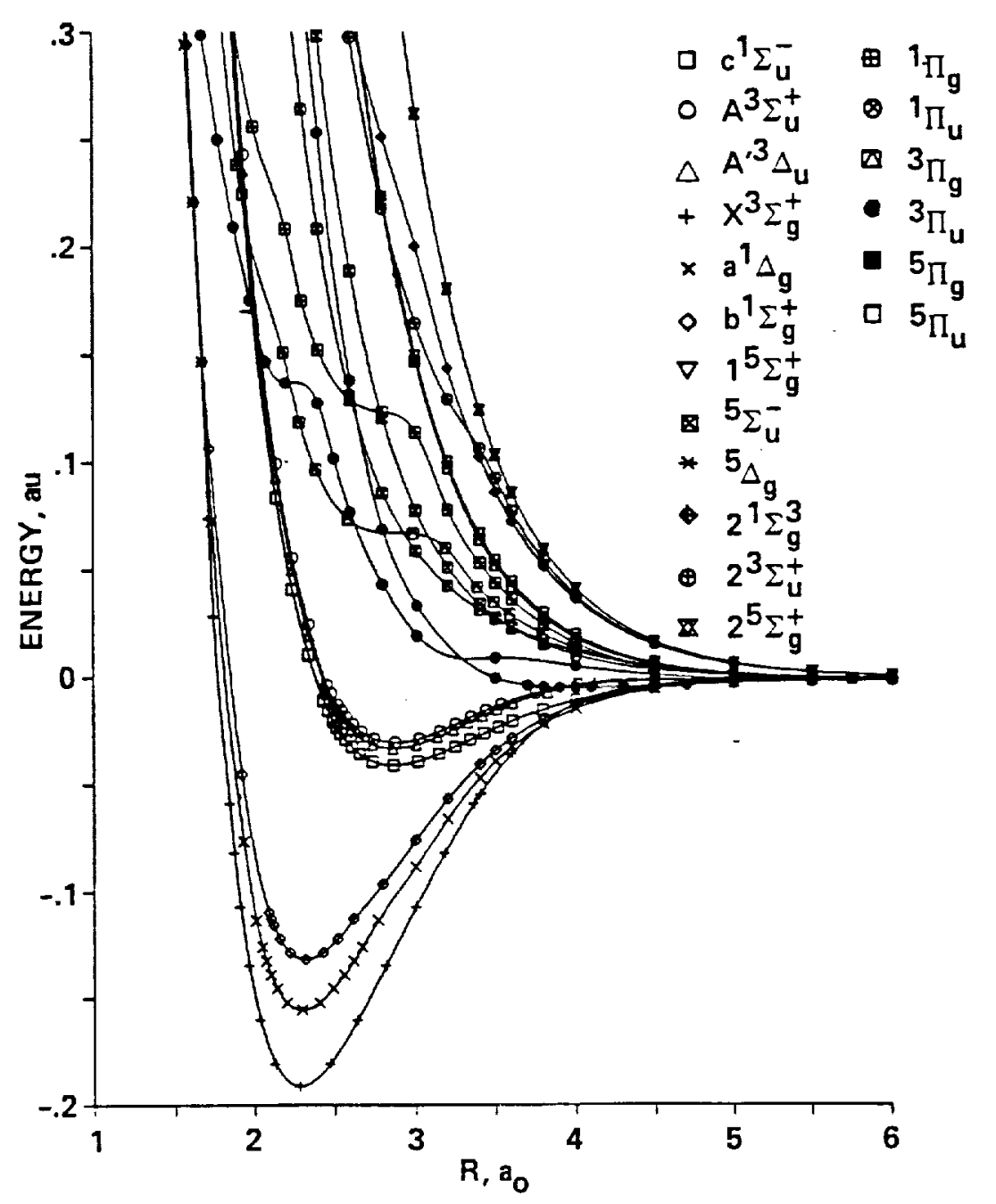

Fig. $2 \mathrm{O}_{2}$ potential energy curves. 


$$
V_{e}(r)=V(r)+\frac{E b^{2}}{r^{2}}
$$

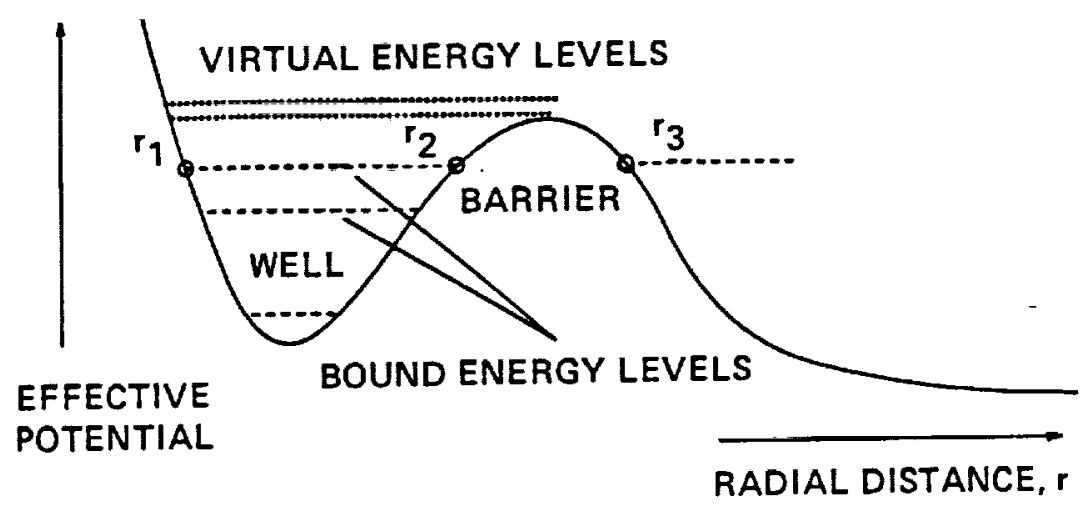

Fig. 1 Diagram for scattering by potential energy barrier. 